\title{
Bloodstream Infection Risk, Incidence, and Deaths for Hospitalized Patients during Coronavirus Disease Pandemic
}

\author{
Bhavarth S. Shukla, Prem R. Warde, Eric Knott, Sebastian Arenas, Darryl Pronty, Reinaldo Ramirez, \\ Arely Rego, Miriam Levy, Martin Zak, Dipen J. Parekh, Tanira Ferreira, Hayley B. Gershengorn
}

\begin{abstract}
Hospital-acquired infections are emerging major concurrent conditions during the coronavirus disease (COVID-19) pandemic. We conducted a retrospective review of hospitalizations during March-October 2020 of adults tested by reverse transcription PCR for severe acute respiratory syndrome coronavirus 2 . We evaluated associations of COVID-19 diagnosis with risk for laboratory-confirmed bloodstream infections (LCBIs, primary outcome), time to LCBI, and risk for death by using logistic and competing risks regression with adjustment for relevant covariates. A total of 10,848 patients were included in the analysis: $918(8.5 \%)$ were given a diagnosis of COVID-19, and 232 (2.1\%) had LCBIs during their hospitalization. Of these patients, $58(25 \%)$ were classified as having central line-associated bloodstream infections. After adjusting for covariates, COVID-19-positive status was associated with higher risk for LCBI and death. Reinforcement of infection control practices should be implemented in COVID-19 wards, and review of superiority and inferiority ranking methods by National Healthcare Safety Network criteria might be needed.
\end{abstract}

$\mathrm{T}$ he incidence of co-infection with either bacterial or fungal pathogens in patients hospitalized because of coronavirus disease (COVID-19) during the ongoing pandemic has become a topic of great interest. Hospitalized COVID-19 patients have shown coinfection rates as low as $7 \%$ (1) and as high as $15 \%$, and as many as $27 \%$ of those who ultimately die are

Author affiliations: University of Miami Health System, Miami, Florida, USA (B.S. Shukla, P.R. Warde, S. Arenas, D. Pronty, R. Ramirez, A. Rego, M. Levy, D.J. Parekh, T. Ferreira, H.B. Gershengorn); University of Miami Miller School of Medicine, Miami (B.S. Shukla, E. Knott, M. Zak, D.J. Parekh, T. Ferreira, H.B. Gershengorn); Albert Einstein College of Medicine, Bronx, New York, USA (H.B. Gershengorn)

DOI: https://doi.org/10.3201/eid2710.210538 co-infected (1-5). Although some COVID-19 patients have bacterial or fungal co-infections, it appears that nosocomial origins for co-infection might be a major factor. One study found that only $3.2 \%$ of hospitalized COVID-19 patients were co-infected at the time of hospital admission (3), and another study demonstrated a cumulative risk of $25 \%$ of developing a bloodstream infection in critically ill COVID-19 patients, but only after 48 hours in the intensive care unit (ICU) (6).

Sparse evidence exists that directly compares nosocomial incidence of bloodstream infection in those having COVID-19 with other hospitalized populations. A multicenter study in New York, New York, USA, found bloodstream infections in only $3.8 \%$ of hospitalized patients who had COVID-19 but in $8.0 \%$ of patients who did not have COVID-19 (7). When comparing with patients who had influenza, Hughes et al. found a 1.8-fold increased rate of bloodstream infection in COVID-19 patients (2.5\% vs. $1.4 \%)$ hospitalized in the United Kingdom (3). However, differences in the types of case-patients by COVID-19 status were not considered in either study. Moreover, the generalizability of these differences by COVID-19 status to other geographic regions remains unknown.

Little evidence exists for risk factors for nosocomial infection in COVID-19. A single-center study from Wuhan, China, identified an association related to use of invasive devices and combination antimicrobial drugs, as well as having diabetes mellitus, with an increased risk for developing a hospital-acquired infection (HAI) (8). However, the external validity of these associations has not been explored.

In this study, we sought to investigate whether being infected with COVID-19 was independently associated with an increase in odds of developing a 
laboratory-confirmed bloodstream infection (LCBI). We also aimed to identify other potential risk factors for LCBI in hospitalized COVID-19 patients. We hypothesized that COVID-19 patients would have greater odds of acquiring an LCBI than hospitalized patients without COVID-19 after adjusting for relevant confounders, and that other risk factors might also be identified, which might serve as targets for interventions to reduce co-infection rates in this vulnerable group.

\section{Methods}

\section{Study Design and Cohort}

We conducted a retrospective cohort study of adult hospitalizations during March 25-October 27, 2020, at an academic, tertiary, acute-care facility in Miami, Florida, USA, which lacks capacity to give care with extracorporeal membrane oxygenation. Patients were included in the cohort if they had $\geq 1$ reverse transcription PCR completed; patients could be included more than once if they were admitted to the hospital more than once over the study period. During the study period, all patients were screened by reverse transcription PCR before hospital admission. Although there were no specific exclusion criteria, the facility does not offer pediatric or obstetric services, so pregnant woman and patients $<18$ years of age were not included. A restricted cohort of patients that had central venous catheters at any point during their hospital stay was also considered.

\section{Exposure and Outcomes}

Our exposure of interest was COVID-19 positivity (determined by SARS-CoV-2 PCR testing) during the hospital stay. Patients who had $\geq 1$ positive test result (from 7 days before admission up until discharge with or without preceding negative test results) were considered positive for COVID-19. Our primary outcome was LCBI. Secondary outcomes were death, time to LCBI (time from hospital admission to first positive blood culture per patient admission), and development of central line-associated bloodstream infection (CLABSI) evaluated by using the restricted cohort of patients who had a central venous catheter. We defined LCBIs and CLABSIs according to National Healthcare Safety Network (NHSN) 2020 criteria (9). In brief, LCBI is defined in these criteria as a single positive blood culture or molecular test result for a pathogen or 2 positive blood cultures for a commensal organism. CLABSI is defined as an LCBI associated with a central venous catheter in place for $\geq 2$ calendar days (9).

\section{Data Sources and Variables}

We obtained information for each patient from the hospital system's electronic medical record by using EPIC software (https://www.epic.com). In addition to COVID-19 infection status and outcomes (including organism identification), we abstracted information on demographics (age, sex, race, primary insurance provider), organisms isolated from blood cultures, chronic health conditions, Elixhauser comorbidity conditions (10), body mass index, severity of acute illness (sequential organ failure assessment score [SOFA] during hospital day 1) (11), renal replacement therapy (either intermittent or continuous), invasive mechanical ventilation, care in the ICU, prone positioning (including persons using mechanical ventilation), central venous catheters, urinary catheters, systemic corticosteroids, tocilizumab, and remdesivir. For each of the resources other than prone positioning, we identified whether they were used and the total duration of use. For prone positioning, we were able to identify only use (not duration or timing of use). We also obtained data on hospital length of stay.

\section{Statistical Analysis and Ethics}

We described the cohort by using standard summary statistics. We made compared characteristics by outcome by using $\chi^{2}$ and Kruskal-Wallis tests as appropriate. Our primary analysis was a risk for LCBI assessment by using multivariable logistic regression modeling with an exposure of COVID-19 status. We included all data elements except prone positioning, remdesivir, and tocilizumab as covariables, and resource elements were modeled as receipt/nonreceipt before development of LCBI (or hospital discharge if no LCBI). To ensure our results would not be confounded by deaths in hospitals, we recreated the same models for hospital survivors and decedents separately.

To consider secondary outcomes, we first used multivariable Cox proportional hazards modeling with censoring at hospital discharge and a competing risk for death to assess the association of COVID-19 positivity and time to LCBI. We then constructed a multivariable logistic regression model to assess the association of COVID-19 positivity with risk for death by hospital discharge. For this model, we included days of resource use as covariates.

We also constructed 3 models to evaluate for LCBI that developed $\geq 2$ calendar days from admission, indicated as LCBI HAI. Next, we reconstructed the models (for our primary and 2 secondary outcomes) by using the restricted cohort of patients who had used central venous catheters to assess risk for and time to 
CLABSI and death. Finally, to identify risk factors for infection among COVID-19 patients, we constructed 3 multivariable logistic regression models: for LCBI among all COVID-19 patients, for LCBI HAI among all COVID-19 patients, and for CLABSI among COVID-19 patients who had central venous catheters.

Because of a large number of patients who had missing data regarding calculation of SOFA, we imputed this score for each model (using multivariable regression modeling, including all covariates and outcome). We conducted 2 sensitivity analyses to assess the robustness of our primary analysis: only patients with an available SOFA score, and all patients but not including SOFA as a model covariate.

Study approval was obtained from the University of Miami Institutional Review Board (\#20200739). We performed all analyses by using the programming language R (R Foundation for Statistical Computing, https://www.r-project.org). Results were considered significant if $p<0.05$. Because we did not adjust for multiple comparisons, we considered all nonprimary analyses to be hypothesis generating.

Table 1. Patients characteristics by outcome on bloodstream infection risk, incidence, and deaths for hospitalized patients during coronavirus disease pandemic, Miami, Florida, USA, March 25-October 27, 2020*

\begin{tabular}{|c|c|c|c|c|c|c|c|c|c|}
\hline \multirow[b]{3}{*}{ Characteristic } & \multicolumn{6}{|c|}{ Full cohort for LCBI analyses, no. (\%) } & \multirow{2}{*}{\multicolumn{3}{|c|}{$\begin{array}{c}\text { Central line cohort for CLABSI } \\
\text { analyses, no. (\%) }\end{array}$}} \\
\hline & \multicolumn{3}{|c|}{ NHSN LCBI } & \multicolumn{3}{|c|}{ NHSN LCBI (HAI) } & & & \\
\hline & No LCBI & LCBI & $\mathrm{p}$ value† & No LCBI & LCBI & $\mathrm{p}$ value & No CLABSI & CLABSI & $\mathrm{p}$ value \\
\hline Patient admissions & $\begin{array}{l}10,616 \\
(97.9)\end{array}$ & $\begin{array}{l}232 \\
(2.1)\end{array}$ & & $\begin{array}{l}10,694 \\
(98.6)\end{array}$ & $\begin{array}{c}154 \\
(1.4)\end{array}$ & & $\begin{array}{l}2,840 \\
(98.0)\end{array}$ & $\begin{array}{c}58 \\
(2.0)\end{array}$ & \\
\hline $\begin{array}{l}\text { COVID-19 RT-PCR } \\
\text { positive }\end{array}$ & $854(8.0)$ & $64(28)$ & $<0.001$ & $857(8.0)$ & $61(40)$ & $<0.001$ & $365(13)$ & $32(55)$ & $<0.001$ \\
\hline Age, y & $\begin{array}{c}63 \\
(52-73)\end{array}$ & $\begin{array}{c}66 \\
(55-74)\end{array}$ & 0.093 & $\begin{array}{c}63 \\
(52-73)\end{array}$ & $\begin{array}{c}66 \\
(54-74)\end{array}$ & 0.4 & $\begin{array}{c}66 \\
(55-76)\end{array}$ & $\begin{array}{c}66 \\
(56-72)\end{array}$ & 0.3 \\
\hline Sex‡ & & & 0.094 & & & 0.001 & & & 0.016 \\
\hline $\begin{array}{l}M \\
F\end{array}$ & $\begin{array}{l}5,479(52) \\
5,136(48)\end{array}$ & $\begin{array}{l}134(58) \\
98(42)\end{array}$ & 0.094 & $\begin{array}{l}5,512(52) \\
5,181(48)\end{array}$ & $\begin{array}{l}101(66) \\
53(34)\end{array}$ & 0.001 & $\begin{array}{l}1,343(47) \\
1,497(53)\end{array}$ & $\begin{array}{l}37(64) \\
21(36)\end{array}$ & 0.016 \\
\hline Race/ethnicity & & & 0.041 & & & 0.053 & & & $<0.001$ \\
\hline Non-Hispanic White & $2,318(22)$ & $33(14)$ & & $2,330(22)$ & $21(14)$ & & $553(19)$ & $7(12)$ & \\
\hline Non-Hispanic Black & $280(2.6)$ & $3(1.3)$ & & $281(2.6)$ & $2(1.3)$ & & $76(2.7)$ & $1(1.7)$ & \\
\hline Hispanic White & $5,152(49)$ & $123(53)$ & & $5,188(49)$ & $87(56)$ & & $1,338(47)$ & $33(57)$ & \\
\hline Hispanic Black & $1,952(18)$ & $49(21)$ & & $1,976(18)$ & $25(16)$ & & $647(23)$ & $4(6.9)$ & \\
\hline Other & $577(5.4)$ & $17(7.3)$ & & $581(5.4)$ & $13(8.4)$ & & $154(5.4)$ & $8(14)$ & \\
\hline Unknown & $337(3.2)$ & $7(3.0)$ & & $338(3.2)$ & $6(3.9)$ & & $72(2.5)$ & $5(8.6)$ & \\
\hline Payer & & & $<0.001$ & & & $<0.001$ & & & 0.077 \\
\hline Commercial & $3,849(36)$ & $59(25)$ & & $3,869(36)$ & $39(25)$ & & $741(26)$ & $15(26)$ & \\
\hline Government & $73(0.7)$ & $4(1.7)$ & & $74(0.7)$ & $3(1.9)$ & & $17(0.6)$ & $2(3.4)$ & \\
\hline Medicaid & $1,248(12)$ & 35 (15) & & $1,254(12)$ & 29 (19) & & 407 (14) & $12(21)$ & \\
\hline Medicare & $4,964(47)$ & $130(56)$ & & $5,014(47)$ & $80(52)$ & & $1,597(56)$ & $27(47)$ & \\
\hline Other & $482(4.5)$ & $4(1.7)$ & & $483(4.5)$ & $3(1.9)$ & & $78(2.7)$ & $2(3.4)$ & \\
\hline BMI, $\mathrm{kg} / \mathrm{m}^{2} \S$ & $\begin{array}{c}27 \\
(23-31) \\
\end{array}$ & $\begin{array}{c}26 \\
(23-30)\end{array}$ & 0.2 & $\begin{array}{c}27 \\
(23-31)\end{array}$ & $\begin{array}{c}27 \\
(23-31)\end{array}$ & 0.7 & $\begin{array}{c}26 \\
(22-31)\end{array}$ & $\begin{array}{c}28 \\
(25-33) \\
\end{array}$ & 0.028 \\
\hline $\begin{array}{l}\text { Elixhauser comorbidity } \\
\text { index }\end{array}$ & $\begin{array}{c}15 \\
(4-29)\end{array}$ & $\begin{array}{c}27 \\
(15-40)\end{array}$ & $<0.001$ & $\begin{array}{c}15 \\
(4-29)\end{array}$ & $\begin{array}{c}26 \\
(15-39)\end{array}$ & $<0.001$ & $\begin{array}{c}23 \\
(11-36)\end{array}$ & $\begin{array}{c}22 \\
(12-34)\end{array}$ & $>0.9$ \\
\hline Urethral catheter & $3,406(32)$ & $126(54)$ & $<0.001$ & $3,430(32)$ & $102(66)$ & $<0.001$ & $1,249(44)$ & $45(78)$ & $<0.001$ \\
\hline Central line & $2,690(25)$ & $208(90)$ & $<0.001$ & $2,754(26)$ & $144(94)$ & $<0.001$ & NA & NA & NA \\
\hline Mechanical ventilation & $750(7.1)$ & $97(42)$ & $<0.001$ & $767(7.2)$ & $80(52)$ & $<0.001$ & $569(20)$ & $39(67)$ & $<0.001$ \\
\hline Steroid treatment & $3,094(29)$ & $127(55)$ & $<0.001$ & $3,119(29)$ & $102(66)$ & $<0.001$ & $1,155(41)$ & $49(84)$ & $<0.001$ \\
\hline ICU admission & $2,043(19)$ & $135(58)$ & $<0.001$ & $2,067(19)$ & $111(72)$ & $<0.001$ & $1,118(39)$ & $48(83)$ & $<0.001$ \\
\hline Dialysis & $657(6.2)$ & $82(35)$ & $<0.001$ & $682(6.4)$ & $57(37)$ & $<0.001$ & $312(11)$ & $29(50)$ & $<0.001$ \\
\hline SOFA scoreף & $1(0-3)$ & $3(2-5)$ & $<0.001$ & $1(0-3)$ & $3(2-5)$ & $<0.001$ & $2(1-4)$ & $3(2-4)$ & 0.055 \\
\hline Central line duration, $d$ & $\begin{array}{c}0.0 \\
(0.0-0.3)\end{array}$ & $\begin{array}{c}14.2 \\
(5.0-28.0 \\
)\end{array}$ & $<0.001$ & $\begin{array}{c}0.0 \\
(0.0-0.4)\end{array}$ & $\begin{array}{c}20.8 \\
(10.2-34 \\
3)\end{array}$ & $<0.001$ & $\begin{array}{c}5 \\
(3-11)\end{array}$ & $\begin{array}{c}28 \\
(15-54)\end{array}$ & $<0.001$ \\
\hline Hospital LOS, d & $\begin{array}{c}3.5 \\
(1.9-6.9)\end{array}$ & $\begin{array}{c}18.8 \\
(9.4-30.9 \\
)\end{array}$ & $<0.001$ & $\begin{array}{c}3.5 \\
(1.9-6.9)\end{array}$ & $\begin{array}{c}24.9 \\
(14.3-36 . \\
7)\end{array}$ & $<0.001$ & $\begin{array}{c}8 \\
(5-14)\end{array}$ & $\begin{array}{c}29 \\
(20-50)\end{array}$ & $<0.001$ \\
\hline Deaths in hospital & $258(2.4)$ & $50(22)$ & $<0.001$ & $267(2.5)$ & $41(27)$ & $<0.001$ & $201(7.1)$ & $21(36)$ & $<0.001$ \\
\hline $\begin{array}{l}\text { *Values are no. (\%) or me } \\
\text { HAl, healthcare associate } \\
\text { NHSN, National Healthca } \\
\text { †Statistical tests perform } \\
\text { †One patient of an unkno } \\
\text { §Eight patients had missi } \\
\text { TIndicates patients who h } \\
\text { cohort } 68(56 \%) \text { of }\end{array}$ & $\begin{array}{l}\text { (IQR). BMI, } \\
\text { ction; IQR, } \\
\text { fety Networ } \\
\text { sher exact t } \\
\text { x in the full } \\
\text { Als in the fu }\end{array}$ & $\begin{array}{l}\text {; Wilcox } \\
\text { hort (did } \\
\text { ohort (3 }\end{array}$ & $\begin{array}{l}\text { k-sun } \\
\text { ave a }\end{array}$ & $\begin{array}{l}\text { ntral line-a } \\
\text { ratory-confi } \\
\text { on PCR; SC } \\
\text { test of ind }\end{array}$ & $\begin{array}{l}\text { d bloods } \\
\text { sequen } \\
\text { ndence. }\end{array}$ & $\begin{array}{l}\text { eam inf } \\
\text { infectio } \\
\text { gan failt }\end{array}$ & $\begin{array}{l}\text { COVID-19, c } \\
\text { length of sta } \\
\text { essment. }\end{array}$ & $\begin{array}{l}\text { navirus di } \\
\mathrm{NA} \text {, not ap }\end{array}$ & plicable; \\
\hline
\end{tabular}



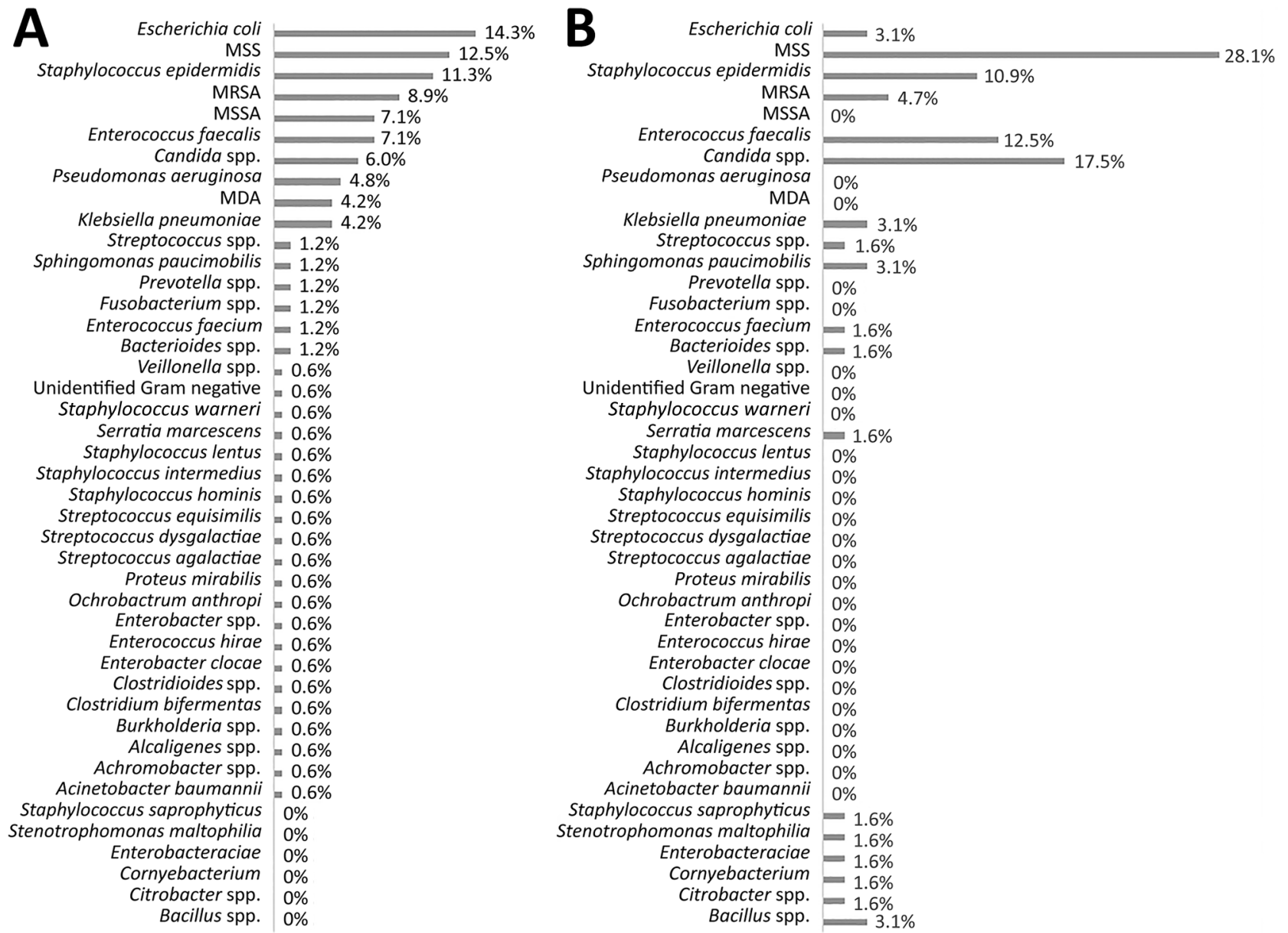

Figure. Organisms responsible for laboratory-confirmed bloodstream infections during COVID-19 pandemic, Miami, Florida, USA, March 25-October 27, 2020. A) COVID-19-negative patients $(n=168)$. B) COVID-19-positive patients $(n=64)$. COVID-19, coronavirus disease; MDA, organisms isolated during admission (defined as $\geq 2$ organisms isolated from the bloodstream $>48$ hours apart during admission); MRSA, methicillin-resistant Staphylococcus aureus; MSSA, methicillin-sensitive S. aureus; MSS, multiple organisms isolated during bloodstream infection episode (defined $\geq 2$ organisms isolated from the bloodstream within a 48 -hour period from the index isolate).

\section{Results}

Our primary cohort consisted of 10,848 hospital admissions, of whom 918 (8.5\%) were COVID-19 positive (Table 1; Appendix Figure 1, https:/ / wwwnc.cdc.gov/EID/article/27/10/21-0538-App1. pdf). A total of 232 (2.1\%) persons showed development of an LCBI: $64(7.0 \%)$ of those who were COVID-19 positive and $168(1.7 \%)$ of those who were COVID-19 negative. The subset of LCBIs acquired 2 calendar days after admission included $61(95.3 \%)$ in the COVID-19-positive patient group and $93(58.4 \%)$ in the COVID-19 negative patient group (Appendix Figure 1). Evaluation of baseline characteristics showed major differences by bivariate analysis of the cohort when divided by outcome (Table 1) or COVID-19 status (Appendix Table 1), including sex, payer, comorbidity index, and SOFA score.
Organisms most frequently cultured meeting NHSN definitions for LCBI among COVID-19 patients were Candida spp. $(\mathrm{n}=11,17.2 \%)$, Enterococcus faecalis $(\mathrm{n}=8,12.5 \%)$, and Staphylococcus epidermidis (7, $10.9 \%)$. These organisms were also found in the context of polymicrobial cultures (internally defined as $\geq 2$ organisms isolated from the bloodstream within a 48 -hour period). They constituted the largest percentage of the cohort of LCBI at $28.1 \%(n=18)$ (Figure). Similar organisms were observed with cultures from COVID-19 patients meeting NHSN definition for CLABSI: Candida spp. $50.0 \%(\mathrm{n}=16)$, E. faecalis $25.0 \%$ $(\mathrm{n}=8)$, and S. epidermis $12.5 \%(\mathrm{n}=4)$. The organisms identified on blood culture from COVID-19-positive versus COVID-19-negative patients for LCBI and CLABSI were comparatively different, but because of low numbers, no statistical analysis was performed (Appendix Figure 2). 
Table 2. Adjusted association for virus positivity with outcomes for patients during the coronavirus disease pandemic, Miami, Florida, USA, March 25-October 27, 2020*

\begin{tabular}{|c|c|c|}
\hline Cohort & Primary outcome & OR/HR $(95 \% \mathrm{Cl})$ \\
\hline \multirow[t]{6}{*}{ Full } & Risk for LCBI† & $3.88(2.70-5.51)$ \\
\hline & Time to LCBI & $2.35(1.77-3.13)$ \\
\hline & $\begin{array}{l}\text { Risk for death,§ } \\
\text { adjusted for LCBI }\end{array}$ & $6.68(4.94-9.01)$ \\
\hline & Risk for LCBI-HAI† & $5.58(3.67-8.43)$ \\
\hline & Time to LCBI-HAI & $2.73(1.94-3.85)$ \\
\hline & $\begin{array}{l}\text { Risk for death, } \S \\
\text { adjusted for LCBI-HAI }\end{array}$ & $6.64(4.91-8.96)$ \\
\hline \multirow[t]{3}{*}{ Central line } & Risk for CLABSI† & $5.68(2.94-11.1)$ \\
\hline & Time to CLABSI & $2.86(1.75-4.65)$ \\
\hline & Risk for death§ & $5.30(3.68-7.64)$ \\
\hline
\end{tabular}

${ }^{*}$ All $p$ values were $<0.001$. BMI, body mass index; CLABSI: central lineassociated bloodstream infection; HR, hazard ratio; ICU, intensive care unit; LCBI, laboratory-confirmed bloodstream infection; OR, odds ratio; SOFA, sequential organ failure assessment.

†Modeled with logistic regression including age, sex, race/ethnicity, payer, BMI, no. comorbidities, previous urethral catheter use, previous central line use, previous mechanical ventilation, previous steroid treatment, previous ICU admission, previous dialysis, previous prone positioning, previous remdesivir treatment, tocilizumab treatment, and imputed SOFA score as covariates. OR is reported.

¥Modeled with proportional hazards model including age, sex, race/ethnicity, payer, BMI, no. comorbidities, previous urethral catheter use, previous central line use, previous mechanical ventilation, previous steroid treatment, previous ICU admission, previous dialysis, and imputed SOFA score as covariates. HR is reported.

\$Modeled with logistic regression including age, sex, race/ethnicity, payer, BMI, no. comorbidities, urethral catheter days, central line days, previous mechanical ventilation days, steroid treatment days, ICU days, dialysis days, imputed SOFA score, and LCBI as covariates; OR is reported.

\section{Association of COVID-19 Status with Outcomes}

After adjusting for potential confounders, we found that COVID-19 positivity was associated with an increase in odds of an LCBI developing (odds ratio [OR] 3.88, 95\% CI 2.70-5.51; $\mathrm{p}<0.001$ (Table 2; Appendix Table 2). COVID-19 was also significantly associated with LCBI developing for either hospital survivors (OR 3.50, 95\% CI 2.28-5.27; $\mathrm{p}<0.001$ ) or decedents (OR 3.14, 95\% CI 1.33-7.72; p = 0.01) (Appendix Table 3) when considered separately. Our results were robust to sensitivity analyses aimed at addressing missing SOFA scores (Appendix Tables 4, 5).

We found significant associations with regards to time to LCBI (hazard ratio 2.35, 95\% CI 1.77-3.13; p <0.001) (Table 2; Appendix Table 2, Figure 3). COVID-19 positivity was associated with an increased odds of hospital death (OR 6.68, 95\% CI 4.94$9.01 ; p<0.001)$. After restricting the cohort to patients with positive cultures after 2 calendar days, we found that COVID-19 was associated with LCBI-HAI; after restricting the cohort to patients with central lines, we found that COVID-19 was associated with CLABSI (Table 2; Appendix Tables 6, 7).

\section{Non-COVID-19 Risk Factors for LCBI, LCBI HAI, and CLABSI}

In a subgroup analysis of only COVID-19 patients, we found that previous central line use was associated with an increased risk for LCBI (OR 8.11, 95\% CI 2.40-37.3; $\mathrm{p}=0.002$ ) and LCBI HAI (OR 11.7, 95\% CI 2.94-78.2; $\mathrm{p}=0.002$ ) (Table 3). We found no major associations with use of remdesivir, steroids, or tocilizumab. Another finding in the subgroup analysis was that prone positioning did not have any major associations with risk for outcomes in patients who had COVID-19.

\section{Discussion}

Before the COVID-19 pandemic, HAIs were well-recognized as a cause of death (12). To date, only a few studies have evaluated the effect of the COVID-19

Table 3. Subgroup analysis of clinical variables in patients who had COVID-19 and bloodstream infection risk, incidence, and deaths for hospitalized patients during coronavirus disease pandemic, Miami, Florida, USA, March 25-October 27, 2020*

\begin{tabular}{|c|c|c|c|c|c|c|}
\hline \multirow[b]{2}{*}{ Characteristic } & \multicolumn{2}{|l|}{$\mathrm{LCBI}$} & \multicolumn{2}{|c|}{ LCBI HAI } & \multicolumn{2}{|l|}{ CLABSI } \\
\hline & OR $(95 \% \mathrm{Cl})$ & $p$ value & OR $(95 \% \mathrm{Cl})$ & $p$ value & OR $(95 \% \mathrm{Cl})$ & $p$ value \\
\hline \multicolumn{7}{|l|}{ Sex } \\
\hline M & $1.84(0.96-3.61)$ & 0.068 & $2.09(1.07-4.23)$ & 0.034 & $3.50(1.29-10.7)$ & 0.019 \\
\hline $\mathrm{F}$ & $0.54(0.28-1.04)$ & 0.068 & $0.48(0.24-0.93)$ & 0.034 & $0.29(0.09-0.77)$ & 0.019 \\
\hline Age, $y$ & $0.97(0.94-1.00)$ & 0.03 & $0.97(0.94-1.00)$ & 0.029 & $0.99(0.94-1.03)$ & 0.6 \\
\hline BMI, $\mathrm{kg} / \mathrm{m}^{2}$ & $0.99(0.94-1.04)$ & 0.7 & $0.99(0.95-1.05)$ & 0.8 & $0.98(0.91-1.05)$ & 0.5 \\
\hline Comorbidity index & $1.00(0.98-1.03)$ & 0.9 & $1.00(0.97-1.02)$ & 0.8 & $0.97(0.93-1.00)$ & 0.094 \\
\hline Previous urethral catheter & $1.96(0.75-5.18)$ & 0.2 & $1.99(0.74-5.41)$ & 0.2 & $1.60(0.28-10.2)$ & 0.6 \\
\hline Previous central line & $8.11(2.40-37.3)$ & 0.002 & $11.7(2.94-78.2)$ & 0.002 & NA & NA \\
\hline Previous mechanical ventilation & $2.82(0.91-9.89)$ & 0.086 & $2.18(0.70-7.44)$ & 0.2 & $\infty(0.00-\infty)$ & $>0.9$ \\
\hline Previous steroid treatment & $0.91(0.40-2.14)$ & 0.8 & $0.91(0.39-2.21)$ & 0.8 & $1.42(0.35-6.73)$ & 0.6 \\
\hline Previous ICU admission & $2.47(0.74-7.61)$ & 0.12 & $3.56(1.07-11.5)$ & 0.034 & $0.00(0.00-\infty)$ & $>0.9$ \\
\hline Previous dialysis & $1.01(0.46-2.14)$ & $>0.9$ & $0.95(0.43-2.07)$ & $>0.9$ & $0.59(0.20-1.68)$ & 0.3 \\
\hline Prone positioning & $1.09(0.49-2.37)$ & 0.8 & $1.21(0.55-2.69)$ & 0.6 & $2.02(0.64-6.97)$ & 0.2 \\
\hline Remdesivir treatment & $1.58(0.78-3.24)$ & 0.2 & $1.58(0.76-3.32)$ & 0.2 & $1.29(0.45-3.77)$ & 0.6 \\
\hline Tocilizumab treatment & $1.29(0.42-3.77)$ & 0.6 & $1.23(0.39-3.62)$ & 0.7 & $1.10(0.25-4.44)$ & 0.9 \\
\hline SOFA score imputed & $1.00(0.86-1.14)$ & $>0.9$ & $0.96(0.82-1.10)$ & 0.6 & $0.083(0.63-1.03)$ & 0.12 \\
\hline
\end{tabular}


pandemic on HAIs and their outcomes, particularly LCBIs $(3,13)$. Using data for $>10,000$ patients hospitalized after SARS-CoV-2 testing, we found that COVID-19 positivity was associated with a 3.88-fold increased odds of developing an LCBI. This finding might be related to COVID-19 itself or other variables not accounted for in our cohort, such as changes in supplementary nursing care or changes in infection control practices associated with the care of these patients. In addition, isolates responsible for LCBI and CLABSI in COVID-19 patients versus non-COVID-19 patients show major differences with regards to type and number of organisms.

Prone positioning has proven benefits for patients who have non-COVID-19-associated acute respiratory distress syndrome requiring invasive mechanical ventilation (14). Studies have noted increases in use of prone positioning as treatment for critical care patients who have influenza (15) and, in recent months, data have emerged suggesting benefits of prone positioning for ventilated patients (16-20) and nonventilated patients who have COVID-19 (21). Although potential adverse effects, such as pressure ulcers (22) and deep venous thromboses (23), have been observed with prone positioning, we did not find any statistical association with our primary outcomes.

The strengths of our study stem from detailed clinical data (including organism identification) and severity of illness information (both acute and chronic) available to us. Our study is limited by a high rate of missing SOFA score data. However, the robustness of our results to sensitivity analyses, in which we excluded patients who had missing SOFA data and excluded SOFA as a model covariate, suggests that this limitation had minimal effect on our findings. Although our sample included $>10,000$ patients (of whom 918 patients had COVID-19), we included only patients from a single hospital, which might limit the generalizability of our results. In addition, several of the factors included in our models occurred after COVID-19 testing (our exposure), making it plausible that these factors are mediators rather than confounders of the association of COVID-19 with outcomes.

Another limitation of the study was our inability to include admission symptoms or central venous catheter insertion sites in the analysis. This limitation was largely caused by inconsistent documentation of these data points in a nondiscrete format in our electronic medical record. We also were not able to address markers of hospital operational stressors that might have varied over the time period of our study and might effect patient outcomes. Collinearity of clinical variables included in the models was also a concern. However, our evaluation identified only 2 variables that had higher correlation coefficients (previous mechanical ventilation and ICU stay) (Appendix Figure 4). A final limitation was the difficulty in analyzing dose and type of steroids and antimicrobial drugs given before and after bloodstream infections, as well as timing and duration of prone positioning.

As more data emerge regarding increases in HAIs during the COVID-19 pandemic (24), we propose that these challenges warrant reevaluation of the NHSN SIR methods for LCBI and CLABSI in COVID-19designated care areas. Further studies are needed to clarify the relationship between COVID-19 and nonLCBI infections to ascertain whether prone positioning, COVID-19-specific treatments, changes in adherence to infection control practices, or a combination of these variables might be associated with higher rates of other HAIs.

In conclusion, inpatient management of patients who have COVID-19 has brought many changes in treatment protocols and associated challenges, including adherence to infection control best practices. Established infection control best practices should be reemphasized among COVID-19 patients who might be at higher risk for LCBI, adding a concurrent condition to an already vulnerable population.

\section{Acknowledgments}

We thank members of the UHealth-Data Analytics Research Team for their support and feedback on this study.

\section{About the Author}

Dr. Shukla is an infectious diseases physician at the University of Miami Miller School of Medicine, Miami, FL. His primary research interest are infection control and hospital epidemiology with a focus on multidrug-resistant bacterial organisms.

\section{References}

1. Lansbury L, Lim B, Baskaran V, Lim WS. Co-infections in people with COVID-19: a systematic review and meta-analysis. J Infect. 2020;81:266-75. https://doi.org/ 10.1016/j.jinf.2020.05.046

2. Wang L, Amin AK, Khanna P, Aali A, McGregor A, Bassett $\mathrm{P}$, et al. An observational cohort study of bacterial co-infection and implications for empirical antibiotic therapy in patients presenting with COVID-19 to hospitals in North West London. J Antimicrob Chemother. 2021;76:796-803.

3. Hughes S, Troise O, Donaldson H, Mughal N, Moore LS. Bacterial and fungal coinfection among hospitalized patients with COVID-19: a retrospective cohort study in a UK secondary-care setting. Clin Microbiol Infect. 2020;26:1395-9. https://doi.org/10.1016/j.cmi.2020.06.025

4. Verweij PE, Gangneux JP, Bassetti M, Brüggemann RJ, Cornely OA, Koehler P, et al.; European Confederation of 
Medical Mycology; International Society for Human and Animal Mycology; European Society for Clinical Microbiology and Infectious Diseases Fungal Infection Study Group; ESCMID Study Group for Infections in Critically Ill Patients. Diagnosing COVID-19-associated pulmonary aspergillosis. Lancet Microbe. 2020;1:e53-5. https:/ / doi.org/10.1016/S2666-5247(20)30027-6

5. Chen N, Zhou M, Dong X, Qu J, Gong F, Han Y, et al. Epidemiological and clinical characteristics of 99 cases of 2019 novel coronavirus pneumonia in Wuhan, China: a descriptive study. Lancet. 2020;395:507-13. https:/ / doi.org/ 10.1016/S0140-6736(20)30211-7

6. Giacobbe DR, Battaglini D, Ball L, Brunetti I, Bruzzone B, Codda G, et al. Bloodstream infections in critically ill patients with COVID-19. Eur J Clin Invest. 2020;50:e13319. https://doi.org/10.1111/eci.13319

7. Sepulveda J, Westblade LF, Whittier S, Satlin MJ, Greendyke WG, Aaron JG, et al. Bacteremia and blood culture utilization during COVID-19 surge in New York City. J Clin Microbiol. 2020;58:e00875-20. https:/ / doi.org/ 10.1128/JCM.00875-20

8. He Y, Li W, Wang Z, Chen H, Tian L, Liu D. Nosocomial infection among patients with COVID-19: A retrospective data analysis of 918 cases from a single center in Wuhan, China. Infect Control Hosp Epidemiol. 2020;41:982-3. https://doi.org/10.1017/ice.2020.126

9. National Healthcare Safety Network (NHSN). Patient safety component manual [cited2020 Oct 13]. https://www.cdc. gov/nhsn/pdfs/pscmanual/pcsmanual_current.pdf

10. Elixhauser A, Steiner C, Harris DR, Coffey RM. Comorbidity measures for use with administrative data. Med Care. 1998;36:8-27. https:// doi.org/10.1097/ 00005650-199801000-00004

11. Vincent JL, Moreno R, Takala J, Willatts S, De Mendonça A, Bruining $\mathrm{H}$, et al. The SOFA (sepsis-related organ failure assessment) score to describe organ dysfunction/failure. On behalf of the Working Group on Sepsis-Related Problems of the European Society of Intensive Care Medicine. Intensive Care Med. 1996;22:707-10. https://doi.org/10.1007/BF01709751

12. Umscheid CA, Mitchell MD, Doshi JA, Agarwal R, Williams K, Brennan PJ. Estimating the proportion of healthcare-associated infections that are reasonably preventable and the related mortality and costs. Infect Control Hosp Epidemiol. 2011;32:101-14. https:/ / doi.org/ 10.1086/657912

13. Kokkoris S, Papachatzakis I, Gavrielatou E, Ntaidou T, Ischaki E, Malachias S, et al. ICU-acquired bloodstream infections in critically ill patients with COVID-19.

J Hosp Infect. 2021;107:95-7. https:/ / doi.org/10.1016/ j.jhin.2020.11.009

14. Guérin C, Reignier J, Richard JC, Beuret P, Gacouin A, Boulain T, et al.; PROSEVA Study Group. Prone positioning in severe acute respiratory distress syndrome. N Engl J Med. 2013;368:2159-68. https://doi.org/10.1056/NEJMoa1214103
15. Marin-Corral J, Climent C, Muñoz R, Samper M, Dot I, Vilà C, et al.; en representacion del Grupo de Trabajo H1N1 GETGAG SEMICYUC. Patients with influenza A (H1N1) pdm09 admitted to the ICU. Impact of the recommendations of the SEMICYUC. Med Intensiva (Engl Ed). 2018;42:473-81. https://doi.org/10.1016/j.medine.2018.08.002

16. Weiss TT, Cerda F, Scott JB, Kaur R, Sungurlu S, Mirza SH, et al. Prone positioning for patients intubated for severe acute respiratory distress syndrome (ARDS) secondary to COVID-19: a retrospective observational cohort study. Br J Anaesth. 2021;126:48-55. https:/ / doi.org/10.1016/ j.bja.2020.09.042

17. Gleissman H, Forsgren A, Andersson E, Lindqvist E, Lipka Falck A, Cronhjort M, et al. Prone positioning in mechanically ventilated patients with severe acute respiratory distress syndrome and coronavirus disease 2019. Acta Anaesthesiol Scand. 2020.

18. Berrill M. Evaluation of oxygenation in 129 proning sessions in 34 mechanically ventilated COVID-19 patients. J Intensive Care Med. 2021;36:229-32. https://doi.org/ 10.1177/0885066620955137

19. Shelhamer MC, Wesson PD, Solari IL, Jensen DL, Steele WA, Dimitrov VG, et al. Prone positioning in moderate to severe acute respiratory distress syndrome due to COVID-19: a cohort study and analysis of physiology. J Intensive Care Med. 2021;36:241-52. https:/ / doi.org/10.1177/0885066620980399

20. Khullar R, Shah S, Singh G, Bae J, Gattu R, Jain S, et al. Effects of prone ventilation on oxygenation, inflammation, and lung infiltrates in COVID-19 related acute respiratory distress syndrome: a retrospective cohort study. J Clin Med. 2020;9:4129. https:// doi.org/10.3390/jcm9124129

21. Venus K, Munshi L, Fralick M. Prone positioning for patients with hypoxic respiratory failure related to COVID-19. CMAJ. 2020;192:E1532-7. https:/ / doi.org/10.1503/cmaj.201201

22. Munshi L, Del Sorbo L, Adhikari NK, Hodgson CL, Wunsch H, Meade MO, et al. Prone position for acute respiratory distress syndrome. A systematic review and meta-analysis. Ann Am Thorac Soc. 2017;14(Suppl 4):S280-8. https:/ / doi.org/10.1513/AnnalsATS.201704-343OT

23. Klok FA, Kruip MJ, van der Meer NJ, Arbous MS, Gommers DA, Kant KM, et al. Incidence of thrombotic complications in critically ill ICU patients with COVID-19. Thromb Res. 2020;191:145-7. https://doi.org/10.1016/ j.thromres.2020.04.013

24. Patel PR, Weiner-Lastinger LM, Dudeck MA, Fike LV, Kuhar DT, Edwards JR, et al. Impact of COVID-19 pandemic on central-line-associated bloodstream infections during the early months of 2020, National Healthcare Safety Network. Infect Control Hosp Epidemiol. 2021;Mar 15:1-4.

Address for correspondence: Bhavarth S. Shukla, University of Miami Health System, 1321 NW 14th St, Ste 602, West Bldg, Miami, FL 33136, USA; email: bxs729@miami.edu 